\title{
Interrupción Escolar y Arreglos Familiares: Aportes a los Estudios de Deserción Escolar Desde el Análisis de la Relación Familia-Escuela
}

Inés Olivera Rodríguez

Pontificia Universidad Católica del Perú

Inés Olivera Rodríguez es Magíster en Ciencias de la Educación y Licenciada en Antropología con especialización en estudios de género. Es docente de cursos de metodología de investigación cualitativa en la especialdiad de antropología de la Pontificia Universidad Católica del Perú. Tiene experiencia de trabajo como investigadora en el área de educación, concentrándose en temas vinculados con el estudio de la relación familiaescuela y exclusión social a través de un enfoque micro-social. Sus temas de interés son el campesinado, juventud, género y metodología de investigación cualitativa.

El presente artículo ha sido elaborado en base al tercer capítulo de mi tesis de maestría, la que desarrollé entre los años 2006 y 2008 en el Programa de Estudios de Postgrado del Departamento de Ciencias de la Educación de la Universidad Federal de Santa Catalina en Brasil. Estos estudios y, asimismo, la tesis, fueron financiados y posibilitados por la beca integral que me fuera otorgada por el Consejo Nacional de Desarrollo Científico y Tecnológico (CNPq), dependiente del Ministerio de Ciencia y Tecnología de Brasil. Agradezco al CNPq por el financiamiento, y a mi asesora Nadir Zago por su acompañamiento y aporte a lo largo de la elaboración de la tesis, y a los y las habitantes de Chaquira por su tiempo, afecto, comprensión y disponibilidad. Agradezco también a Santiago Cueto cuyos ricos y detallados comentarios permitieron trasformar lo que fuera un capítulo de tesis en un artículo independiente y capaz de traer y sustentar una discusión relevante para el análisis de la realidad educativa nacional. Finalmente, agradezco también a Danilo de Assis Clímaco quien es responsable de la traducción del portugués al castellano del capítulo que sirvió de base para el presente artículo y, como siempre, por el placer de aprender juntos. 


\title{
Interrupción Escolar y Arreglos Familiares: Aportes a los Estudios de Deserción Escolar Desde el Análisis de la Relación Familia-Escuela
}

\begin{abstract}
Resumen
La literatura sobre exclusión social e inequidad en los estudios sobre educación ha evidenciado, desde diferentes puntos de análisis, cómo los sectores populares son excluidos del sistema de diversas formas, estando la mayoría de las explicaciones asociada a las condiciones económicas de las familias. No obstante, estudios de la última década, desde la sociología de la educación, evidencian que, dentro de grupos en similares condiciones económicas, diversos resultados escolares son posibles. En este marco, la pregunta que ocupa al presente estudio es la siguiente: ¿por qué algunos/ as jóvenes que viven en contextos aparentemente similares logran continuar en el sistema escolar mientras otros lo abandonan?
\end{abstract}

Palabras clave: relación estudiante-escuela, educación rural, antropología de la educación, juventud rural

\section{School Interruption and Family Arrangements: Contributions to the Drop-out Literature from the Analysis of Family-School Links}

\begin{abstract}
Studies on social exclusion and inequality within education have shown, from different points of analysis, how working class groups are in fact excluded in diverse ways, with most of the explanations associated with family economic conditions. However, studies in the last decade, from the sociology of education, show that within groups in similar economic conditions, different educational outcomes are possible. In this context, the question that occupies the present study is the following: Why some young men and women living in apparently similar contexts manage to remain in the school system while others leave it?
\end{abstract}

Keywords: school student relationship, rural education, educational anthropology, rural youth 


\section{Introducción}

a literatura sobre exclusión e inequidad en las últimas décadas, empezan-do por los estudios sobre la reproducción social de Bourdieu durante los años setenta, viene evidenciando cómo los grupos populares son expulsados del sistema de diversas formas, siendo una de las principales la presión por trabajo que dificulta la dedicación a los estudios de jóvenes de sectores populares. No obstante, estudios recientes evidencian que, dentro de grupos en similares condiciones de opresión económica, son posibles diversos resultados escolares. La pregunta es, entonces, por qué algunos jóvenes logran seguir en el sistema escolar mientras otros lo abandonan. Se trata de una mirada a la bastante discutida problemática de lo que, por muchos años, se ha llamado deserción escolar.

Un primer aspecto a considerar es que este trabajo parte de la comprensión de que el problema social a ser considerado es el de la interrupción de la escolaridad y no el de la deserción, abandono o fracaso escolar. Estos términos fueron utilizados de forma generalizada y casi sinónima por la literatura sobre el tema durante las décadas pasadas ${ }^{1}$. Como explica Charlot (1996) bajo la lectura de la meritocracia y la igualdad de oportunidades, principios rectores de la escuela moderna y la lectura sociológica que la respal$\mathrm{da}^{2}$, la institución escolar se presenta como neutra e igual para todos/as los/ las miembros de la sociedad. Sin embargo, los estudios de la llamada teoría crítica ${ }^{3}$ en la educación vienen demostrando desde la década de 1960 que las condiciones con las cuales llegan los y las estudiantes a la institución escolar y con las cuales deben lidiar durante su etapa escolar son radicalmente diferentes; y por tanto, la escuela no puede ser verdaderamente meritocrática ni democrática en tanto se piense neutra frente a la sociedad.

Para Charlot, este principio de la escuela moderna meritocrática responsabiliza al individuo por sus resultados escolares de tal forma que la interrupción de sus estudios, la repetición en el sistema o, en términos generales, los resultados escolares no satisfactorios son interpretados como el fracaso del

1 Sobre este punto, puede consultarse, por ejemplo, Carabaña (2002), Parsons (1959), Coleman et al. (1966), Alves (2006) y Carvalho (2003).

2 Véase los textos de Durkheim, por ejemplo, en la compilación Educación y pedagogía publicada en 1998 en Buenos Aires por editorial Losada. 
individuo. De la misma forma, la interrupción de los estudios es interpretada como la voluntad y decisión concreta del individuo que deserta del sistema o lo abandona, nuevamente responsabilizando al mismo por su trayectoria escolar. Esto es cuestionado por algunas corrientes de la teoría crítica como los estudios de Bourdieu y sus seguidores, quienes cuestionan esta responsabilización del individuo y demuestran que es el sistema quien expulsa a ciertas personas, no siendo estas las que "deciden" salir.

A partir de lo señalado, mi propuesta de discutir el tema de la interrupción y no del fracaso escolar se basa en dos reflexiones: en primer lugar, es una crítica frente a una mirada de falsa meritocracia que caracteriza a los estudios liberales ${ }^{4}$ que colocan sobre los sujetos la responsabilidad de su resultado escolar ${ }^{5}$ negando, o evitando ver, la existencia de un sistema social que funciona de tal forma que las diferencias sociales pueden ser reproducidas. Es bajo esta lógica que quienes salen del sistema escolar no son expulsados por el mismo, sino que desertan, fracasan o ambas situaciones a la vez.

En segundo lugar, considero importante evidenciar las limitaciones metodológicas para seguir las trayectorias escolares a gran escala en grupos sociales donde la entrada, salida y retorno al sistema escolar son frecuentes. Encontramos, de esta forma, un campo de especial relevancia para los estudios cualitativos, que nos permiten seguir con mayor detalle las trayectorias escolares de individuos y grupos familiares, sin producir datos falaces y generalizadores de abandono cuando, en realidad, se trata de interrupciones. De esta forma, los estudios que se adscriben a lo que en Brasil se comprende como la sociología de la relación familia-escuela defienden la necesidad de hablar de interrupciones y no de abandono escolar ${ }^{6}$.

El trabajo de campo en el cual se basa el presente artículo fue realizado durante mediados de enero y mediados de marzo de 2007, tiempo durante el cual viví en el lugar de la investigación. Se trató de un trabajo etnográfico que centralizó la observación de las dinámicas juveniles y las entrevistas con un total de 18 jóvenes hombres y mujeres en diferentes situaciones escolares. Son jóvenes campesinos del caserío Chaquira, en la región Piura.

4 Esta crítica está fundamentada en autores como Giroux (1997), McLaren (1995) y Alves (2006).

5 Para mayor detalle sobre esta discusión, puede revisarse el trabajo de Alves (2006), Charlot (1996), Zago (2000) y, por supuesto, Bourdieu y Passeron (2004). zação em camadas médias e populares (Nogueira, 2000). 
Como investigación antropológica, se pretendió una mirada transversal a las diversas instituciones sociales del caserío (es decir, el trabajo, la familia y la escuela), realizando observaciones en los espacios de trabajo agrícola y doméstico, en los escasos espacios recreativos de la juventud y en el colegio secundario.

Posteriormente, se construyeron matrices interpretativas por cada grupo familiar para realizar comparaciones entre grupos familiares: desde la situación económica de las mismas hasta las dinámicas intrafamiliares. En función a esta matriz y considerando los objetivos del presente artículo, fueron seleccionados los ocho casos que permitían una mejor comparación. Los ocho casos del estudio son, entonces, de jóvenes hombres y mujeres que en 2007 tenían entre 15 y 22 años (hoy, entre 19 y 27 años de edad). Estos casos fueron seleccionados a partir de su situación escolar que en 2007 definí dentro de tres posibilidades: escolaridad interrumpida, continuidad escolar o conclusión de la educación básica regular. Para la elaboración del presente texto, en marzo de 2010, retomé el contacto con los ocho casos seleccionados y actualicé la información recogida, centralizando dos situaciones escolares: escolaridad interrumpida y escolaridad concluida, siendo los casos que en la época estaban estudiando en el 2010 o ya concluyeron o interrumpieron los estudios.

\section{Tabla 1}

Los Casos de Análisis

\begin{tabular}{|c|c|c|c|c|c|c|c|}
\hline \multirow{3}{*}{$\begin{array}{l}\text { Situación } \\
\text { escolar }\end{array}$} & \multirow[t]{3}{*}{ Pseudónimo } & \multirow{3}{*}{$\begin{array}{c}\text { Edad al } \\
2010\end{array}$} & \multirow{3}{*}{$\begin{array}{l}\text { Último año } \\
\text { escolar } \\
\text { concluido } \\
\text { al } 2010\end{array}$} & \multicolumn{4}{|c|}{ Trayectorias escolares } \\
\hline & & & & \multicolumn{2}{|c|}{ Reprobaciones } & \multicolumn{2}{|c|}{ Interrupciones ${ }^{*}$} \\
\hline & & & & Primaria & Secundaria & Primaria & Secundaria \\
\hline \multirow{4}{*}{$\begin{array}{l}\text { Casos de } \\
\text { interrupción }\end{array}$} & María & 22 & $4 \mathrm{~s}$ & 1 & 1 & 1 & - \\
\hline & Roberto & 27 & $4 s$ & 3 & 1 & - & - \\
\hline & Omar & 28 & $4 \mathrm{~s}$ & 1 & 0 & - & - \\
\hline & Gisela & 24 & $6 p$ & 1 & - & 1 & - \\
\hline \multirow{4}{*}{$\begin{array}{l}\text { Casos de } \\
\text { conclusión }\end{array}$} & Eloísa & 20 & $5 s$ & 3 & 1 & - & - \\
\hline & Alberto & 18 & $5 s$ & 1 & 0 & - & - \\
\hline & Luis & 27 & $5 s$ & 1 & 0 & - & - \\
\hline & Laura & 22 & $5 s$ & 1 & 0 & - & - \\
\hline
\end{tabular}

* Interrupción entendida como dejar de estudiar durante uno o más años antes de la culminación de la educación básica regular. 


\section{La Existencia del Problema y Relevancia de su Análisis}

Según los indicadores de la educación en el Perú del 2008, producidos por el sistema de estadística de la calidad educativa del Ministerio de Educación del Perú7, el 94.2\% de niños/as de 6 a 11 años de edad a nivel nacional está matriculado en el nivel primario ${ }^{8}$. Según la misma fuente, para el caso del nivel secundario, apenas el $74.8 \%$ de jóvenes a nivel nacional, de 12 a 16 años, está matriculado. Desagregando estas cifras por área geográfica (rural o urbana), encontramos que la tasa neta de matrícula en área urbana cae del $94.2 \%$ en la primaria a $82.5 \%$ en la secundaria, es decir, casi 12 puntos porcentuales. Sin embargo, en el caso del área rural, la caída de la tasa neta de matrícula entre ambos niveles llega a 30 puntos porcentuales; teniendo una tasa neta de matrícula de $94.1 \%$ en la primaria y de $64 \%$ en la secundaria. En el caso específico de la Región Piura, región donde se desarrolló el presente estudio, la tasa neta de cobertura en primaria es de $94 \%$ y en secundaria de $65.3 \%$; lo que quiere decir que, si bien en primaria está dentro del promedio nacional, en secundaria se encuentra diez y siete puntos porcentuales por debajo del promedio nacional. Además, llama la atención que en ambos casos (primaria y secundaria) la tasa neta de matrícula del 2008 sea menor que la registrada para el $2005^{9}$. Si bien es cierto que se trata de caídas porcentuales de menos de un punto, no reflejan el aumento registrado en el promedio nacional en este mismo periodo.

Según la misma fuente, para el 2008, la tasa de deserción escolar a nivel nacional fue de $2.4 \%$ en la primaria y de $6.7 \%$ en la secundaria; lamentablemente, este dato no está desagregado por zona geográfica, pero evidencia que la cobertura y acceso en secundaria representa aún una brecha en comparación con la primaria ${ }^{10}$. El porcentaje de alumnos con atraso escolar resulta también ilustrativo. Este dato considera el porcentaje de estudiantes con una edad mayor a la esperada por el sistema; es decir, estudiantes que han repetido, se

7 Disponible desde Internet en: http://escale.minedu.gob.pe/downloads/indicadores/2008/cuadro. html?C01

8 La cobertura del sistema se calcula en base a la inscripción de estudiantes en el sistema y se contrasta con la información del censo poblacional, que indica el total de niños y niñas de 6 a 11 años de edad en el Perú, considerando estas como las edades "oficiales" de ingreso y salida al nivel primario.

9 La tasa neta de matrícula en la primaria en la región Piura en el 2005 llegó a 95.5\%, y en el 2008, a $94 \%$. En el caso de la secundaria, en el 2005 llegó a $65.4 \%$ y en el 2008 , a $65.3 \%$. html?D05 
han retirado del sistema educativo por uno o más años o ambos casos; o que han ingresado al sistema escolar con una edad mayor a la edad oficial de ingreso al primer grado de primaria (seis años). Según este dato, el atraso escolar de estudiantes a nivel nacional en instituciones educativas públicas está presente en un $18.8 \%$ en la primaria y en un $21.6 \%$ en la secundaria ${ }^{11}$. Al desagregar este dato por zona geográfica, encontramos que el porcentaje de estudiantes con atraso escolar en instituciones públicas urbanas de nivel primario es de $12.3 \%$ y en secundaria es de $17.7 \%$; mientras que, en instituciones públicas rurales, el porcentaje de estudiantes con atraso en nivel primario es $27.2 \%$ y en secundaria llega a $35.6 \%$. Por lo tanto, se duplica el porcentaje de zona urbana en ambos casos. Resulta interesante considerar que, según la misma fuente, es en la primaria cuando los y las estudiantes repiten más, específicamente en segundo y tercer grado de primaria, llegando a más de $15 \%$ de repetidores en estos dos grados. Mientras que, en la secundaria, en ningún grado se registra un porcentaje de repetidores/as mayor al $9 \%^{12}$.

Resulta significativo que, si cruzamos esto último con el dato antes ofrecido, que evidencia una mayor tasa de matrícula en primaria que en secundaria en zona rural, necesitamos buscar explicaciones que nos permitan comprender cómo, si hay menos repeticiones en la secundaria, la tasa de cobertura es menor que en la primaria.

Es frente a esta pregunta que el presente artículo pretende ofrecer la siguiente hipótesis explicativa: los casos analizados parecen evidenciar que, frente a los mayores costos que representa la educación secundaria para las familias, estas deben generar estrategias que les permitan realizar inversiones educativas más racionales para el grupo familiar. Lo que, en la práctica, parece generar la estrategia familiar de evaluación de los desempeños escolares de los hijos e hijas para decidir sobre su continuidad educativa.

Resulta fundamental mencionar que, de todos los recortes realizados para analizar y comparar las tasas de conclusión escolar, tanto en primaria como en secundaria, es en la población rural que se presentan los índices más bajos, siendo apenas mayor al nivel de conclusión en pobres extremos ${ }^{13}$.

11 Disponible desde Internet en: http://escale.minedu.gob.pe/downloads/indicadores/2008/cuadro. html?D03

12 Disponible desde Internet en: http://escale.minedu.gob.pe/downloads/indicadores/2008/cuadro. html?D04. Cuadros D03, D04 y D06 disponibles desde Internet en: http://escale.minedu.gob.pe/ downloads/indicadores/2008/cuadro.html?D03

13 La categoría pobre extremo se refiere a las personas que no pueden pagar una canasta básica de alimentos. 
En otras palabras, la tasa de conclusión de la población rural es menor que la tasa de conclusión de primaria y secundaria de hombres, de mujeres y de población pobre. Esos datos de la estadística nacional revelan, por un lado, la diferencia de cobertura y permanencia entre primaria y secundaria; y por otro, la diferencia entre área rural y área urbana. Todo ello confirma que la continuidad escolar es un problema en el Perú, sobre todo, en las áreas rurales y, principalmente, para los jóvenes de 12 a 16 años $^{14}$, que frecuentan o deberían frecuentar la secundaria.

\section{La Propuesta Interpretativa}

Frente a la evidencia de la estadística y de la realidad nacional, el presente artículo tiene por objetivo discutir los límites de la explicación de la interrupción de la escolaridad en los jóvenes rurales por su condición de trabajadores. Para desarrollar esta cuestión, me apoyo en los trabajos empíricos (Diez, 1998; Olivera, 2009; Ansión, 1989) que evidencian la existencia de un fuerte vínculo entre los sectores populares y la escuela, confrontando la literatura que atribuye a la condición de trabajadores el motivo de la interrupción escolar (Montero, 1995).

Evidentemente, la condición de trabajadores coloca a los y las jóvenes de sectores populares en desventaja frente a los y las jóvenes que no tienen que lidiar con condiciones de opresión económica, pudiendo dedicarse al estudio de forma exclusiva. Sin embargo, estudios realizados desde la década de 1990 vienen mostrando diferencias en los resultados escolares de grupos socioeconómicos en condiciones similares ${ }^{15}$. Esto quiere decir que el trabajo no imposibilita radicalmente la continuidad escolar y que, en determinadas condiciones, es posible para algunos/as jóvenes combinar trabajo y estudio ${ }^{16}$.

Si bien se trata de analizar en profundidad las trayectorias y motivos de la situación escolar de los y las jóvenes, se parte desde una comprensión de la familia campesina como grupo integrado que comparte objetivos comunes y se organiza para lograrlos. Los estudios sobre población campesina en el país

Edades oficiales de los y las estudiantes de secundaria para el Ministerio de Educación. Se puede consultar, por ejemplo, Lahire (1997), Olivera (2008) y Dayrell (1996).

16 Sobre ese punto, se puede consultar el interesante libro de Guedes (1997) sobre los encuentros y desencuentros de trabajo y estudio en la formación de jóvenes trabajadores. 
muestran el principio colectivista de esta sociedad, según el cual la familia nuclear es comprendida como un grupo integrado que desarrolla estrategias para la producción y reproducción de la sociedad rura ${ }^{17}$. Así, para comprender las relaciones de los jóvenes del campo con la escuela rural en el Perú, es necesario incluir un análisis de esas estrategias familiares.

La investigación de Lahire (1997) sobre los diversos arreglos familiares en torno a la organización de los recursos que potencializan o limitan las posibilidades de continuidad escolar es fundamental para un análisis como el propuesto aquí. El autor investigó el comportamiento de niños, niñas y sus familias en la periferia parisiense, analizando los arreglos en la organización familiar del trabajo y de los mecanismos familiares de socialización, a partir de cinco ejes: "las formas familiares de la cultura escrita, las condiciones y disposiciones económicas, el orden moral doméstico, las formas de autoridad familiar, y las formas familiares de inversión pedagógica" (p. 20). La propuesta del autor es que, al analizarse esos aspectos, es posible identificar no solo el lugar de la escolaridad en los proyectos familiares, sino también el lugar que ocupa la cultura escolar en el cotidiano familiar de los niños y niñas, y la construcción, consciente o no, de estrategias que permitan maximizar los recursos familiares, económicos y humanos para la continuidad escolar.

No obstante, cabe señalar que reconocer la prolongación de la escolaridad y los esfuerzos que las familias de sectores populares desarrollan para conseguirla no niega que los y las jóvenes que logran culminar la escolaridad obligatoria se confrontan con un sistema de desigualdad que limita la prolongación de los estudios. Es decir, el análisis que se presenta comprende el sistema social y escolar como un sistema de reproducción de las diferencias sociales como el descrito por Bourdieu y Passeron (1998). Sin embargo, el aporte está en la evidencia de la diversidad presente dentro de los procesos de exclusión social.

En este estudio, la propuesta analítica de Lahire (1997) es reformulada en los elementos propuestos para el análisis, a la luz de la realidad rural de la costa norte y de las familias campesinas peruanas. Un estudio previo evidenció para el caso de Chaquira que, si bien es cierto que las familias no priorizan el estudio de los hijos mayores o menores, o el de los hijos hombres por sobre el de las hijas mujeres, el lugar que ocupan los hijos en el grupo de hermanos tiende a tener repercusión en las oportunidades escolares que tienen los y las 
jóvenes del caserío (Olivera, 2005). Esto, como se demostrará en el presente texto, básicamente se debe a la relación tierra familiar - mano de obra familiar disponible ${ }^{18}$.

Es relevante analizar, entonces, el espacio de negociación entre las diversas actividades realizadas por los jóvenes que pueden influir en su disponibilidad para los estudios. En el caso de Chaquira, trabajos anteriores evidenciaron que las diferentes cargas de trabajo que tienen los y las jóvenes, que son relativamente independientes de su edad y sexo, determinan las oportunidades de continuidad escolar. Es decir, si bien existen trabajos asumidos como femeninos o masculinos y trabajos realizados a partir de cierta edad, se identifica, en el plano empírico, que estas normas son adaptadas en cada grupo familiar. Así, no solo por ser tema central en las discusiones sobre deserción escolar (Zago, 2000), aquí considerado como interrupción de la escolaridad, sino también por su relevancia concreta para el análisis del caso en cuestión, se identifica el criterio económico familiar como central para analizar las particularidades de los arreglos familiares.

Un aspecto también importante en la literatura sobre deserción escolar es el vinculado al capital escolar familiar, como lo plantean Lareau, (2002), Mickelson (2003) y Epstein (2005). Estas autoras, que utilizan el concepto de los capitales de Bourdieu (específicamente, el escolar), sostienen que niños y niñas con padres "más educados" tienen mayores posibilidades de tener éxito en la escuela. Esto se debe a diversos motivos: porque cuentan con mayor apoyo de los padres y madres en su proceso escolar, porque existen discursos positivos sobre la escuela en la familia, porque hay una familiaridad con la cultura escolar (específicamente, se presenta el ejemplo de la lectura), y todo esto vendría a repercutir, además, en una mayor disponibilidad de los padres de familia a realizar gastos en la educación de sus hijos. Aunque podríamos comenzar por discutir la noción misma de "más educados", es importante considerar los resultados de los estudios que demuestran que más importante que lo económico (principalmente, en sociedades donde la educación pública es, al menos en teoría, gratuita) parece ser los discursos positivos sobre la escuela.

A partir de esto, definí los siguientes cuatro criterios para analizar los "arreglos familiares" de las familias campesinas de Chaquira: las características

18 Este tema fue trabajado posteriormente con mayor detalle en mi tesis de maestría al identificar las diferencias en las oportunidades escolares asociadas a los recursos económicos de jóvenes dentro de un mismo núcleo familiar (Olivera, 2008). 
del grupo de hermanos o fratría, la distribución del trabajo en el interior del grupo familiar, las condiciones y disposiciones económicas, y las experiencias escolares del grupo familiar.

Conceptos también extraídos de la teoría crítica de la educación, como curriculum oculto, permiten identificar los puntos de conflicto entre la institución escolar y los/las estudiantes. Por otro lado, aún partiendo de la noción de estrategias familiares, no se puede negar que los y las propias estudiantes o posibles estudiantes tienen voz en los procesos de decisión de continuidad escolar. Este aspecto es también central en el análisis de los casos que se presentan en este trabajo una vez que la primera explicación de algunos jóvenes frente a la pregunta por los motivos de su interrupción es "ya no quise estudiar". Un análisis profundo de sus trayectorias escolares y de las descripciones de las situaciones que los llevaron a tomar esta decisión, arroja evidencias de la conflictiva relación con la escuela. En las décadas pasadas, el concepto de curriculum oculto ha servido para los análisis desde las categorías de género y raza en la educación ${ }^{19}$, así como para los estudios sobre democracia en la escuela ${ }^{20}$ y las, llamadas por Giroux (1997), lecturas críticas radicales de la educación. Sin hacer referencia a este concepto, pero centralizando los conflictos sociedad-escuela, los trabajos de Thin (2006) evidencian los procesos de imposición de un tipo de conocimiento y valoración de una cultura específica por parte de la escuela que crea distanciamiento entre ella y las familias de sectores populares.

Finalmente, consideramos importante para la interpretación de los casos utilizar la noción de mito de la escuela, tan discutida en la investigación educativa del Perú, acuñada por los primeros estudios sobre educación desde la antropología (Ansión, 1989; Degregori, 1986; Montoya, 1980). La noción de mito de la escuela hace referencia a la forma como se interpretó la extensa acogida de la escuela por parte de la población campesina en el Perú desde la década de 1960. El mito de la escuela hace referencia a la necesidad de la población andina de acceder al saber occidentalizado para lograr insertarse en la sociedad mayor que excluye su cultura. Algunos investigadores cuestionan la utilidad explicativa actual del mito de la escuela. Lamentablemente, estos cuestionamientos no han sido formulados en discusiones teóricas, empíricas o conjuntas al respecto. Frente a esto, trabajos empíricos recientes continúan encontrando referencias al valor de la escolaridad en el campo que pueden explicarse por la noción del mito de la escuela (Olivera, 2009).

19 Véase, por ejemplo, Rosemberg (2001) y Carvalho (2003).

20 Véase, por ejemplo, Reimers y Villegas-Reimers (2006). 


\section{Capital Escolar Familiar y Conflictos con la Escuela: Buscando Explicaciones a los Vínculos Frágiles con la Escolaridad más allá de lo Económico}

Roberto, Gisela, María y Omar componen el grupo de jóvenes que interrumpió su escolaridad. En ese grupo, los/las cuatro entrevistados/as participan del trabajo familiar de forma permanente, sea con su fuerza de trabajo o con parte de sus salarios. Así, María, de 22 años e hija mayor, se dedica de forma exclusiva al cuidado de la familia (padres y hermanos/as) y Omar, de 28 años, trabaja en la agricultura familiar; en tanto que los otros dos entrevistados, Roberto de 27 y Gisela de 24, aportan a la economía familiar con su salario.

Roberto es el quinto de ocho hermanos/as, el tercero de los hombres, e inició su participación en la economía familiar a los 20 años. Eran sus hermanos mayores quienes trabajaban el pequeño terreno agrícola familiar (de 0,5 hectáreas) y, por estar estudiando, él participaba en la agricultura familiar solo de forma eventual, lo que, aún así, lo obligó a faltar algunas veces a la escuela. Dentro de su grupo familiar, Roberto es el único con estudios de secundaria. Todos sus hermanos y hermanas llegaron a tercero de primaria, su madre tiene primer grado, y su padre no tiene experiencia escolar alguna.

Cuando Roberto interrumpió los estudios y necesitó insertarse en el trabajo agrícola familiar, los hermanos mayores y los menores ya estaban trabajando en ella. Claramente, la presión por trabajo no fue el motivo de la interrupción de la escolaridad para él. De esta forma, entre el momento en que interrumpió su escolaridad, a los 18 años, y el inicio en el trabajo asalariado, a los 20, Roberto permaneció desempleado. Fue solo la inserción en el trabajo asalariado lo que le permitió contribuir a la economía familiar.

Roberto afirma haber interrumpido su escolaridad por "decisión personal" al finalizar el penúltimo año de secundaria. En su testimonio sobre ese proceso, dice que habría optado por la interrupción debido a una situación de conflicto con una profesora que lo habría castigado por un motivo que consideraba injusto:

Me gritó y dijo que me iba a suspender... yo le trataba de explicar que había tenido que faltar porque tenía que ayudar a mi papá en la parcela... que había sido cosa de unos días nada más y que sí había estudiado para esa clase. Ella comenzó con eso de que no me importaba, y que mis 
papás también no se importaban con mi estudio. Ahí yo me sentí mal, porque era bien raro que yo faltara, y no era por gusto, solo cuando era pues necesario. (Roberto, 27 años).

Él contó, posteriormente, que en vista de que no regresaba al colegio, el director lo llamó para conversar y que otra profesora lo había buscado para convencerlo de retomar los estudios, pero él no quiso. Su frustración ante la situación de injusticia vivida en la escuela habría sido tan grande que eliminó toda posibilidad de retorno. Este caso es ilustrativo debido a que, al menos aparentemente, Roberto tiene posibilidades económicas y familiares de continuidad escolar, no obstante la relación con la escuela al ser frágil no motiva lo suficiente a Roberto para invertir en ella (Dubet \& Martuccelli, 1998). El sentido de la escolaridad que habría movilizado a Roberto por tantos años parece ya no tener ese efecto.

Pero, más allá de la fragilidad del vínculo con la escuela, en ese caso, se observa otro proceso. Los propios mecanismos excluyentes en el interior de la escuela, de cierta forma, habrían empujado a Roberto fuera del sistema. Esto, considerando que según la Directiva para el Año Escolar 2007, "en el área rural se establece el horario de la jornada diaria que mejor se adecue a la realidad local" (Ministerio de Educación [MED], 2006, p. 8). Así, la propia legislación establece que, para que los estudiantes del campo no pierdan clases debido a su participación en el trabajo familiar, la escuela debe adaptarse a los horarios de la organización local. Según Roberto, "si la escuela funcionara por las tardes, no tendría que faltar nunca".

Se observa, en el caso de Roberto, la concretización de la disonancia entre las formas de socialización escolar y familiar (Thin, 2006), donde el trabajo familiar es percibido por la escuela como irresponsabilidad de estudiantes y familias que no priorizan la asistencia a la escuela. La escuela reclama falta de compromiso de los estudiantes y de sus familias, mientras los estudiantes reclaman falta de reconocimiento de su esfuerzo. Sobre esta base, sostengo que, cuando este choque entre las lógicas de socialización escolar y familiar es el protagonista, la relación con la escuela corre el riesgo de perder sentido. Al llevar a los estudiantes del campo ante el límite de sus posibilidades exigiendo condiciones imposibles como su no participación en el trabajo familiar, la escuela pierde su legitimidad ante la juventud.

El caso de Gisela es diferente, ya que, hasta el momento de su salida de la vivienda familiar, fue ella la principal responsable de los trabajos de cuidado, siendo la mayor de una fratría conformada por cuatro mujeres y dos hombres 
(los menores). En el grupo familiar de Gisela, el padre cuenta con primaria completa y la madre con segundo grado; Gisela y dos de sus hermanas tienen primaria completa; el mayor de los varones se encuentra, en el 2010, realizando estudios de secundaria; y el menor, comenzando la primaria. La segunda en el grupo de hermanos es la única de las mujeres que llegó a secundaria, interrumpió sus estudios en cuarto año y, hasta el momento, no la ha retomado.

Al contrario de Roberto, desde temprano, Gisela vivía en una situación de sobre-ocupación. Ella era responsable por la totalidad de los trabajos de cuidados en el hogar, incluyendo la preparación y venta de chicha para la generación de ingresos. Gisela tuvo que interrumpir su escolaridad al terminar la primaria, lo que, al contrario de Roberto, habría ocurrido contra su voluntad. Según lo que ella y su madre recuerdan, se quejó mucho por esta decisión de sus padres:

Al comienzo no me conformaba... yo gritaba, me desesperaba, les decía a mis papás que me dejaran estudiar... Les preguntaba que por qué mi hermana sí podía seguir estudiando y yo no... Pero nada, ellos decían que mi mamá estaba enferma, que tenía que entender y que hacia falta que yo me encargue de la casa... (Gisela, 24 años).

Si bien Gisela era la mayor, tenía ya experiencias de reprobación escolar, y fue por este motivo, según afirma ella misma, que recayó la responsabilidad doméstica sobre ella. Es importante aquí remarcar el hecho de que, aún siendo explícito su deseo de seguir estudiando, las condiciones familiares no le dieron posibilidades de negociación. La estrategia en la familia habría sido, tras una evaluación de los desempeños escolares, atribuir a una de las hijas los trabajos de cuidado con el objetivo de garantizar la continuidad escolar de las/os otras/os. Continuó así hasta los cuatro años siguientes. La segunda de las hijas cursaba el cuarto de secundaria cuando la economía familiar se vio más comprometida por el embarazo de la madre, del cual nacería el sexto hermano. Frente a esta situación, se evaluó la necesidad de aumentar los ingresos de la familia, enviando a una de las hijas a la ciudad para trabajar. Esta vez, Gisela reclamó esta posibilidad para sí, argumentando ya haber sido perjudicada anteriormente en beneficio de las hermanas cuando interrumpió su escolaridad. Manifestó que era su "turno" de acceder a lo que consideraba la mejor opción: migrar y ya no cargar con los trabajos familiares de cuidado. La migración surgió, así, como una compensación a la interrupción de la escolaridad. 
De esta forma, si para Roberto el trabajo asalariado fue el mecanismo de inserción en el universo de trabajo, para Gisela el trabajo fue siempre una demanda por lo que la migración vino a representar una disminución de la misma. Hasta el 2009, por la proximidad de su lugar de trabajo, mantenía el vínculo con la familia visitándola todo los fines de semana. Además, contribuía con la economía familiar, no con la entrega de una parte de su sueldo, sino asumiendo los costos de la escolaridad del único hermano que seguía estudiando y del tratamiento de la madre que tenía problemas crónicos de salud. Con el dinero restante, Gisela realizaba gastos personales, saliendo con las amigas y visitando Internet. En el 2009, Gisela se casó y tuvo un hijo; se mudó al centro poblado mayor Casa Grande (cercano a Chaquira); y desde entonces, se dedica al cuidado de su casa y de su hijo. Su esposo es funcionario del Municipio.

María tiene hoy 22 años y es la mayor de tres hermanas. Sus padres llegaron a segundo grado de primaria; ella interrumpió sus estudios al concluir el tercero de secundaria; la segunda de sus hermanas, al concluir el primero de secundaria; y la tercera se encuentra, actualmente, en tercero de secundaria. De esta forma, así como en los casos de Roberto y Gisela, María no contaba con un referente de escolaridad prolongada dentro de su familia nuclear.

María interrumpió su escolaridad por haber reprobado algunos cursos al final del tercero de secundaria. Según ella, su deseo, como el de los padres, era el de seguir estudiando, pero la familia no logró cubrir los costos de los exámenes de recuperación ${ }^{21}$. Estos exámenes tienen un costo de 10 nuevos soles cada uno. Dado que la familia no pudo pagarlos, María no pudo ser promovida de año, y el colegio tampoco ofreció una alternativa. Considerando la existencia de la propiedad agrícola familiar y su dimensión como criterios de diferenciación socioeconómica dentro del caserío, la familia de María forma parte del $21.59 \%$ de familias no propietarias agrícolas. Por lo tanto, está entre las más pobres por carecer de fuentes de producción y ocupación de mano de obra masculina. Por ese motivo, el padre de María se encuadra en la categoría trabajador agrícola asalariado eventual, que depende de la familia extensa ${ }^{22}$ para emplearse y conseguir una fuente de renta.

21 El mismo director del Ricardo Palma confirma que, cuando algún o alguna estudiante es reprobado/a en un curso, debe someterse a un examen al final del verano, para lo cual la escuela cobra diez nuevos soles.

22 La familia extensa es parte fundamental de la organización de las familias andinas y campesinas del Perú. El término hace referencia a los vínculos de consaguinidad, afinidad e espiritualidad (padrinos, por ejemplo) que vinculan las personas más allá de la familia nuclear. Para las familias andinas, la familia extensa mantiene obligaciones de reciprocidad y cooperación. 
Aparentemente, en este caso, es la imposibilidad de pago el motivo de la interrupción, evidenciándose los mecanismos de reproducción de la escuela que, siendo pública y gratuita por derecho constitucional, incurre en cobros ilegales que dificultan la permanencia en el sistema escolar de jóvenes de sectores populares. No obstante, un análisis más profundo del caso de María permitirá identificar otros factores que influyen en ese proceso. María recuerda que, por ser la hija mayor, tuvo que comenzar a ayudar a su madre con los trabajos de casa desde los 12 años, en el mismo año en que empezó los estudios de secundaria. Por ese motivo, debía organizarse para realizar algunos trabajos domésticos en la mañana temprano antes de salir rumbo a la escuela, y otros durante la tarde. Cuenta que aunque

...sí hacía las tareas cuando ya me desocupaba por las tardes, pero así no más lo que habían pedido, no me daba tiempo para estudiar. Así leer mis libros, mis cuadernos, no me alcanzaba el tiempo... (Gisela, 24 años).

De esa forma, si las dificultades económicas impidieron el pago de los exámenes de recuperación, la falta de tiempo para el estudio fue una dificultad previa, probablemente decisiva, para la continuidad escolar de María. Puedo afirmar, entonces, que en casos como los de María, el problema se perfila más allá de las condiciones económicas, ya que un mayor presupuesto familiar no habría librado a María de la responsabilidad de asumir buena parte de los trabajos de cuidado. La organización familiar del trabajo corresponde a un sistema cultural y a un proceso de socialización en las actividades propias del campo. En la mejor de las situaciones, una mayor capacidad adquisitiva en la familia de María habría posibilitado el pago de los exámenes de recuperación, pero no habría aumentado el tiempo de estudio en la casa.

Omar es el cuarto de un grupo de once hermanos, y es el mayor de los varones. Su familia tiene una parcela pequeña de tierra, de una hectárea, por lo que Omar logró cursar hasta cuarto de secundaria sin mayor exigencia de trabajo. Sin embargo, la enfermedad de su madre generó presión sobre la economía familiar, lo que llevó a que Omar decida interrumpir los estudios para buscar un empleo asalariado en la ciudad de Piura y enviar dinero a su familia. Omar no logró conseguir empleo en la ciudad y volvió a Chaquira, donde se ocupó como jornalero con otras familias. Así, comenzó a colaborar con la economía familiar. Hasta el momento, no ha retomado los estudios para concluir el último año de secundaria. Omar fue el primero en su familia 


\section{Tabla 2}

Los Casos de Jóvenes que Interrumpieron los Estudios y sus Grupos Familiares

\begin{tabular}{|c|c|c|c|c|c|}
\hline Caso & $\begin{array}{l}\text { Propiedad } \\
\text { agrícola }\end{array}$ & $\begin{array}{l}\text { Rol / lugar } \\
\text { en el grupo } \\
\text { familiar }\end{array}$ & $\begin{array}{l}\text { Edad al } \\
2010\end{array}$ & $\begin{array}{c}\text { Nivel } \\
\text { educativo al } \\
2010\end{array}$ & $\begin{array}{c}\text { Situación escolar } \\
\text { al } 2010\end{array}$ \\
\hline \multirow[t]{5}{*}{ María } & No tiene & Padre & 50 & $2 p$ & Interrumpió \\
\hline & & Madre & 48 & $2 p$ & Interrumpió \\
\hline & & María & 22 & $3 \mathrm{~s}$ & Interrumpió \\
\hline & & Hermana & 21 & $1 \mathrm{~s}$ & Interrumpió \\
\hline & & Hermana & 18 & $3 \mathrm{~s}$ & Continúa \\
\hline \multirow[t]{10}{*}{ Roberto } & 1 Hectárea & Padre & 56 & 0 & No \\
\hline & & Madre & 58 & $1 p$ & Interrumpió \\
\hline & & Hermana & 33 & $3 p$ & Interrumpió \\
\hline & & Hermano & 31 & $3 p$ & Interrumpió \\
\hline & & Hermano & 30 & $3 p$ & Interrumpió \\
\hline & & Hermana & 29 & $3 p$ & Interrumpió \\
\hline & & Roberto & 27 & $4 \mathrm{~s}$ & Interrumpió \\
\hline & & Hermano & 19 & $3 p$ & Interrumpió \\
\hline & & Hermana & 18 & $3 p$ & Interrumpió \\
\hline & & Hermano & 15 & $6 p$ & Continúa \\
\hline \multirow[t]{8}{*}{ Gisela } & 1 Hectárea & Padre & 51 & $6 p$ & Interrumpió \\
\hline & & Madre & 47 & $2 p$ & Interrumpió \\
\hline & & Gisela & 24 & $6 p$ & Interrumpió \\
\hline & & Hermana & 23 & $4 \mathrm{~s}$ & Interrumpió \\
\hline & & Hermana & 21 & $6 p$ & Interrumpió \\
\hline & & Hermana & 18 & $6 p$ & Interrumpió \\
\hline & & Hermano & 16 & $2 \mathrm{~s}$ & Continúa \\
\hline & & Hermano & 6 & 0 & No \\
\hline \multirow[t]{13}{*}{ Omar } & 1 Hectárea & Padre & 60 & 0 & No \\
\hline & & Madre & 53 & 0 & No \\
\hline & & Hermana & 32 & $6 p$ & Interrumpió \\
\hline & & Hermana & 30 & $6 p$ & Interrumpió \\
\hline & & Hermana & 29 & $6 p$ & Interrumpió \\
\hline & & Omar & 28 & $4 \mathrm{~s}$ & Interrumpió \\
\hline & & Hermana & 27 & $6 p$ & Interrumpió \\
\hline & & Hermana & 25 & $5 s$ & Culminó \\
\hline & & Hermano & 24 & $6 p$ & Interrumpió \\
\hline & & Hermana & 21 & $3 \mathrm{~s}$ & Interrumpió \\
\hline & & Hermano & 19 & $6 p$ & Interrumpió \\
\hline & & Hermana & 17 & $6 p$ & Continúa \\
\hline & & Hermana & 13 & $5 p$ & Continúa \\
\hline
\end{tabular}


en culminar la secundaria. Sus tres hermanas menores interrumpieron los estudios al concluir la primaria, y sus padres no cuentan con experiencia escolar. Después de Omar, dos hermanos (una mujer y un hombre) llegaron a secundaria, su hermana concluyó y el hermano interrumpió sus estudios también en tercero. Los últimos dos hermanos son los únicos que continúan estudiando: están por concluir la primaria.

Si bien es una situación imprevisible la que parece explicar la interrupción de la escolaridad de Omar, es interesante notar que la interrupción es un elemento común en la mayor parte de sus hermanos. Esto nos lleva a preguntarnos si se trata de una situación aislada o de la existencia de una relación frágil con la escolaridad para el grupo familiar de Omar.

Intentando englobar lo que tienen en común los cuatro casos de jóvenes que interrumpieron su escolaridad, se percibe que esa situación aparece no solo entre los/las entrevistados/as, sino también entre los otros miembros de sus grupos familiares. Solo dos de los/las cuatro jóvenes tienen un hermano o hermana que ha concluido la secundaria y ninguno de los padres o madres cuenta con secundaria completa; siendo el caso que apenas uno de los padres tiene estudios de secundaria. En estos grupos familiares, el prolongamiento de la escolaridad no parece formar parte de la realidad ni del imaginario familiar.

Por otro lado, considerando la propiedad agrícola como indicador de mayor o menor nivel de pobreza de una familia, ese grupo no podría ser apuntado como el de las familias más pobres, debido a que solo la familia de María no posee propiedad agrícola. Es decir, encontramos diferentes situaciones económicas en familias con escaso capital escolar.

Como se observa en estos cuatro casos, para la juventud de Chaquira frecuentar la secundaria es una situación inestable que puede truncarse en cualquier momento por dificultades financieras, demandas de mano de obra en la familia o tensiones en la interacción entre la escuela y la familia. De esta forma, la ausencia de capital escolar acumulado en el núcleo familiar, y el distanciamiento entre la escuela y la realidad local parecen ser dos elementos centrales.

\section{Contextos Favorables y Arreglos Familiares: Buscando Recurrencias en los Casos de Continuidad y Conclusión Escolar}

Eloísa, Alberto, Laura y Luis componen el grupo de jóvenes que, en el 2007, cuando fue realizado el trabajo de campo, estaba estudiando o ya había 
culminado la secundaria. Para el presente año 2010, los dos aún estudiantes de la época han concluido la secundaria. En este grupo de jóvenes, al contrario del grupo anterior, tanto las mujeres como los hombres jóvenes tienen como principal ocupación el estudio.

En lo referente a las chicas, Eloísa, hoy con 20 años, y Laura, hoy con 22; sus grupos familiares se organizan de tal forma que permiten liberarlas lo máximo posible de los trabajos domésticos para garantizar su mejor desempeño escolar. Y esto se puede apreciar no solamente en sus casos específicos, sino también en la recurrencia de mujeres con experiencia escolar prolongada en sus grupos familiares. Se evidencia la existencia de una estrategia familiar pensada para maximizar las posibilidades de continuidad escolar de uno de sus miembros. Esta situación demuestra, empíricamente y en otro contexto, las afirmaciones de Zoomers (1998) sobre la organización de estrategias familiares campesinas en la persecución de objetivos comunes.

Eloísa es la cuarta de una fratría de cinco y la tercera de las mujeres. Sin embargo, sus dos hermanas mayores (la segunda y tercera en el grupo de hermanos) migraron a Lima para trabajar, lo que dejó a Eloísa como la única hija que puede ayudar a la madre en los trabajos de cuidado. Frente a los casos anteriormente analizados, cabe formularse la siguiente pregunta: ¿qué ocurre en esta familia para que Eloísa, siendo la principal fuente de apoyo para el trabajo doméstico, pueda dedicarse mañanas y tardes al estudio? Se trata de la confluencia de situaciones externas a la familia, que posibilitan determinados arreglos y decisiones familiares. Así, Eloísa es responsable por algunos trabajos de cuidado, pequeños y puntuales, participando eventualmente en el trabajo de la casa.

Se trata de una reorganización del trabajo familiar con el objetivo de aumentar el tiempo que Eloísa dispone para estudiar. Esto es posible en la medida en que tres de los cinco hijos no son más responsabilidad de ese grupo familiar; la madre se encarga de casi la totalidad de los trabajos de cuidado, así como de la preparación y venta de chicha; y el hermano menor la ayuda con algunos de los trabajos que comúnmente son desarrollados por las hijas. De esta forma, es por el ajuste de los proyectos a las condiciones materiales, que la familia de Eloísa encamina sus acciones y decisiones buscando la continuidad de la formación escolar de los/las hijos/as.

La familia de Eloísa es propietaria agrícola: el padre y el hermano mayor trabajan en la parcela familiar. Además de eso, su padre es propietario de un pequeño camión que utiliza para el transporte, aumentando la renta familiar. 
Esto, junto con las contribuciones de las hijas que trabajan fuera, permite una situación económica relativamente estable, lo que, sumado a la alta valorización de la escolaridad por parte de la familia, propicia que la continuidad escolar de los hijos no sea cuestionada. Esto se observa en las trayectorias escolares de los integrantes del grupo, en el cual dos de los tres hermanos mayores concluyeron la escolaridad obligatoria, mientras que la tercera la interrumpió tras el tercer año de la secundaria. La escolaridad prolongada se presenta, incluso, en la trayectoria del padre que tiene también secundaria completa.

El tercer caso de este grupo de jóvenes estudiantes es el de Alberto, hoy con 18 años y el mayor en una fratría de cuatro. Su familia no tiene propiedad agrícola ni otra fuente de empleo propia. Su padre trabaja como albañil de forma eventual, por lo que familia se sustenta prácticamente con el dinero semanal de la venta de chicha. En lo que respecta a los trabajos de cuidado, él afirma que son sus dos hermanas, hoy con 17 y 14 años, quienes ayudan a la madre. En este caso, las propias condiciones familiares de escasez de tierra agrícola aparecen como "posibilitando" la continuidad escolar de Alberto, puesto que no se le exige participar del trabajo agrícola.

Los últimos dos casos de este grupo se refieren a una misma familia. Se trata de Luis, hoy con 27 años, y Laura, hoy con 22, que son el sexto y la octava, respectivamente, de un grupo de nueve hermanos. Este grupo de hermanos está compuesto por siete mujeres y dos hombres. Aunque ninguno de los padres cuenta con primaria completa, la escolaridad prolongada es una situación común en el grupo de hermanos. Las tres hijas mayores interrumpieron los estudios en primaria. Ellas cuentan que fue por presiones económicas. Además, las tres tuvieron períodos de trabajo como empleadas domésticas en Lima. Sin embargo, de la cuarta a la novena de las hijas, incluyendo los dos varones, tienen todos secundaria completa. La cuarta de las hijas inició estudios de enfermería que no concluyó; y Laura, la penúltima, está por concluir sus estudios de secretariado en un instituto técnico superior en Lima.

Luis, Laura y Vilma, la quinta de los hermanos, viven en Lima. Los tres trabajan en fábricas: Luis y Vilma se desempeñan como obreros en procesos de producción textil, mientras que Laura es ayudante en la oficina de administración en la misma fábrica donde trabaja Vilma. En un primer momento, tanto Laura como Vilma se desempeñaron como empleadas domésticas.

Como Laura recuerda sobre su época de escolar, cuando ella y su her- 
habían independizado, motivo por el cual, si bien es cierto que eran las menores, fueron la única fuente de apoyo posible para la madre. Esto parece haber generado un nivel de tensión entre sus padres por lo que, según Laura:

...mi mamá era quien renegaba. Decía a veces nos decía, para qué tanto estudio y yo sola nadie me ayuda. Pero mi papá sí nos defendía y le decía que nos deje estudiar que era lo único que nos iban a dejar... (Laura, 22 años).

Así, en esta familia, la posibilidad de estudio de Laura se debió a una centralización de los trabajos de cuidado en la madre para que las hijas puedan estudiar. Como mencionaba líneas arriba, las escolaridades prolongadas son comunes en este grupo familiar. En el caso de las hermanas mayores, lo que permitió la culminación de los estudios fue el apoyo económico enviado desde Lima por las hermanas que se fueron a trabajar. Por lo tanto, se identifica la organización del trabajo y de los recursos económicos para aumentar los tiempos de estudios de las y los hijos.

El caso de su hermano Luis es bastante similar: ni él ni su hermano Carlos ayudaron al padre en el trabajo agrícola cuando eran jóvenes. Ambos se dedicaban de forma exclusiva a los estudios. Como para sus hermanas mujeres, esto fue posible debido el apoyo económico de las hermanas que trabajaban en Lima, lo que permitía al padre pagar jornales para ayudarse en la producción agrícola familiar.

Sin desconsiderar las complicaciones del empleo informal, resulta fundamental reconocer que, para la juventud de Chaquira, existe en la ciudad una oferta ocupacional a la cual puede tener acceso, satisfaciendo la principal motivación de la migración: obtener trabajo asalariado que permita ahorrar y ayudar a la familia.

Sostengo que esa misma informalidad ocupacional explica por qué muchos jóvenes retornan aunque el objetivo sea establecerse definitivamente en la ciudad. Al no tener acceso al mercado de empleo formal, resulta difícilmente concretizable la migración permanente, que exige una estabilidad económica mínima. Por otro lado, mientras dura, la migración permite enviar dinero a la familia, invertir en la propia persona e incluso ahorrar dinero. En otras palabras, aun cuando es temporal, la migración permite ascender a mejores condiciones de vida en términos materiales y financieros. 


\section{Tabla 3}

Los Casos de Jóvenes que Concluyeron los Estudios y sus Grupos Familiares

\begin{tabular}{|c|c|c|c|c|c|}
\hline Caso & $\begin{array}{l}\text { Propiedad } \\
\text { agrícola }\end{array}$ & $\begin{array}{l}\text { Rol / lugar en el } \\
\text { grupo familiar }\end{array}$ & $\begin{array}{c}\text { Edad al } \\
2010\end{array}$ & $\begin{array}{c}\text { Nivel } \\
\text { educativo al } \\
2010\end{array}$ & $\begin{array}{c}\text { Situación escolar } \\
\text { al } 2010\end{array}$ \\
\hline \multirow[t]{6}{*}{ Alberto } & \multirow[t]{6}{*}{ No tienen } & Padre & 40 & $3 s$ & Interrumpió \\
\hline & & Madre & 41 & $2 p$ & Interrumpió \\
\hline & & Alberto & 18 & $4 \mathrm{~s}$ & Culminó \\
\hline & & Hermana & 17 & $3 \mathrm{~s}$ & Culminó \\
\hline & & Hermano & 14 & $4 p$ & Continúa \\
\hline & & Hermana & 12 & $2 p$ & Continúa \\
\hline \multirow[t]{7}{*}{ Eloísa } & \multirow{7}{*}{$\begin{array}{l}1,25 \\
\text { hectáreas }\end{array}$} & Padre & 50 & $5 s$ & Culminó \\
\hline & & Madre & 54 & $2 p$ & Interrumpió \\
\hline & & Hermano & 26 & $5 s$ & Culminó \\
\hline & & Hermana & 24 & $5 s$ & Culminó \\
\hline & & Hermana & 22 & $3 \mathrm{~s}$ & Interrumpió \\
\hline & & Eloísa & 20 & $5 s$ & Culminó \\
\hline & & Hermano & 17 & $2 \mathrm{~s}$ & Interrumpió \\
\hline Luis y & \multirow{11}{*}{$\begin{array}{l}1 \\
\text { hectárea }\end{array}$} & Padre & 70 & $2 p$ & Interrumpió \\
\hline \multirow[t]{10}{*}{ Laura } & & Madre & 56 & $1 p$ & Interrumpió \\
\hline & & Hermana & 39 & $5 p$ & Interrumpió \\
\hline & & Hermana & 37 & $6 p$ & Interrumpió \\
\hline & & Hermana & 36 & $5 p$ & Interrumpió \\
\hline & & Hermana & 35 & ES & Culminó \\
\hline & & Hermano & 33 & $5 s$ & Culminó \\
\hline & & Luis & 27 & $5 s$ & Culminó \\
\hline & & Hermana & 26 & $5 s$ & Culminó \\
\hline & & Laura & 22 & $5 s$ & Culminó \\
\hline & & Hermana & 20 & $5 s$ & Culminó \\
\hline
\end{tabular}

Apuntando las recurrencias en este grupo de jóvenes, en comparación con el anterior, se nota que las experiencias escolares familiares son más prolongadas, por tanto una mayor acumulación de capital escolar. Todos tienen hermanos, padres o ambos con escolaridad completa. Cabe resaltar que son los padres y nunca las madres quienes tienen escolaridad prolongada. Esto evidencia un proceso de cambio en el caserío en el cual, si en las generaciones anteriores la escolaridad era exclusiva de los hombres, en las actuales la escolaridad beneficia indistintamente estudiantes hombres y mujeres.

Finalmente, un aspecto fundamental que caracteriza este grupo tiene que ver con la organización del trabajo familiar, como es evidente en el caso de Eloísa, Laura y Luis. El caso de Alberto muestra, más bien, situaciones en 
las que, si no estuvieran estudiando, estarían desempleados, sea porque las familias no tienen tierra para cultivar o porque tienen más hijos de los que pueden emplear. De esa forma, se evidencia que la continuidad escolar de estos jóvenes no tiene relación directa con una mejor posición económica dentro del caserío. Por el contrario, en este grupo de jóvenes, se concentran familias con propiedades agrícolas muy pequeñas o sin propiedad alguna, diferenciándose del grupo de jóvenes que interrumpió sus estudios. La ampliación de la escolaridad, para estos casos, parece tener relación con una menor demanda familiar por el trabajo de los/las hijos/as en los trabajos de la familia y no con mejores condiciones económicas.

Así, la principal diferencia respecto al grupo anterior sería que, ante la incapacidad de la escuela para adaptarse al entorno local, las familias que no necesitan o que tienen dificultades de emplear todos/as los/las hijos/as en la propiedad familiar, tienen condiciones de mantenerlos dedicados exclusivamente a los estudios. Evidentemente, se trata de situaciones en que es requerido mucho compromiso por parte de los estudiantes, puesto que las economías familiares no permiten asumir costos adicionales, como pagar los exámenes de recuperación.

\section{Los Aportes de la Descripción Antropológica para el Análisis de los Motivos de Interrupción Escolar en el Perú}

Como vienen demostrando los estudios antropológicos, un análisis microsocial permite identificar las sutilezas de las desigualdades en espacios aparentemente iguales, así como aporta a la relativización del mandato de las condiciones económicas como únicos elementos y, por lo tanto, determinantes de la inclusión o exclusión social. La pobreza y la exclusión, así como el desarrollo y el bienestar, están lejos de ser cuestiones simples: exigen reconocer patrones culturales y a las organizaciones y estrategias que los grupos humanos desarrollan para alcanzar sus objetivos. Dentro de ese tipo de análisis, la descripción densa como recurso de investigación antropológica evidencia que es en los detalles de la vida cotidiana que se configuran las desigualdades. Esa fue la propuesta y justificación para presentar la información a partir de la complejidad de los arreglos familiares, en vistas a hacer evidente para los/las lectores/as que son varios los elementos que, combinados, posibilitan o limitan la continuidad escolar de los/las jóvenes que inician sus proyectos de vida a partir de condiciones adversas. 
A continuación, discuto algunas de las explicaciones dadas por la literatura especializada sobre los motivos de interrupción de la escolaridad en sectores populares. Estas son, básicamente, de dos tipos: las que hacen hincapié en los motivos económicos y los estudios que, desde diferentes preguntas, analizan el rol de la escolaridad en el imaginario de desarrollo de las personas.

Los arreglos familiares presentados evidencian, justamente, que las diferencias económicas de las familias en Chaquira son mínimas y que, cuando existen, no son suficientes para explicar la interrupción o continuidad escolar. De los casos analizados, tanto los jóvenes que culminaron como los que interrumpieron la escolaridad tienen las mismas dimensiones de propiedad agrícola. La diferencia, veíamos, tiene que ver con la demanda concreta de trabajo que tienen los jóvenes. $Y$ esto depende del equilibrio entre tierra disponible y mano de obra con las cuales cuenta una familia. De esta forma, vimos que, en algunos casos, para los hijos varones menores resulta más probable continuar estudiando cuando sus hermanos ya están empleados en la parcela familiar y que, por tanto, no solo no había demanda por su trabajo, sino que, además, no había posibilidad de emplearlo. De esta forma, como discutía al inicio de este trabajo, no se trata del lugar de los hijos en la fratría, sino de la organización del trabajo frente a la necesidad de mano de obra concreta en un grupo familiar.

En esta misma línea, veíamos en los casos analizados que, en general, la demanda por trabajo femenino es mayor que la que existe por trabajo masculino; afirmábamos que esto tiene que ver con la escasez de tierra agrícola. Sin embargo, encontramos que la continuidad escolar no se explica por un factor de género, ya que, en términos generales, en Chaquira las mujeres culminan la secundaria tanto como los hombres y, como se ve en los casos presentados y en sus grupos familiares, las mujeres permanecen en el sistema escolar. Lo que ocurre aquí es, entonces, lo que hemos definido como la organización del trabajo familiar, es decir, la creación de estrategias para reorganizar los trabajos de cuidado de tal forma que las mujeres puedan continuar estudiando. En algunos casos, implica que la madre lleva a cabo todas las tareas; en otros, ha significado que los hijos varones menores realicen algunas tareas generalmente definidas como femeninas, como ayudar en la cocina; y finalmente, en otras situaciones, significa colocar más responsabilidades sobre las estudiantes mujeres que están en la primaria para liberar a las hijas que llegaron a la secundaria. En estos casos, se evidencia el despliegue de una estrategia conciente que tiene por objetivo aumentar las posibilidades de continuidad escolar de las mujeres. Estrategia que implica, además, una apuesta colectiva de "sacrificio" no solo de parte de padres, sino también de parte de hermanos y hermanas. 
Entra aquí la segunda explicación, y es la relacionada con el papel que juega la escolaridad en los proyectos de vida de las personas. La pregunta es qué hace que algunas familias estén dispuestas a generar estrategias para aumentar las posibilidades de los hijos, o las de algunos de ellos/ellas, y otras no. Es aquí donde trabajos como los de Lareau (2002) y otros hablan de la importancia del capital escolar familiar. La idea es que los padres con experiencias escolares prolongadas tienden a invertir más en la educación de sus hijos. En los casos analizados, encontramos que, efectivamente, familias en similares condiciones económicas y con similares estructuras familiares tienen hijos con históricos escolares diversos. Si bien es cierto que en los casos de análisis el nivel de escolaridad de padres y madres no parece ser diferente en los casos de continuidad o interrupción, lo que sí se encuentra es que los grupos familiares de jóvenes que concluyeron o continúan estudiando en secundaria no son casos aislados; es decir, la escolaridad prolongada es recurrente en el grupo de hermanos. Podemos, por lo tanto, pensar en otra forma de capital escolar familiar, no de padres a hijos pero sí entre hermanos.

Más que un cierre, este análisis pretende provocar alguna reflexión en torno de la continuidad escolar a partir de lo que la escuela significa en términos de beneficios para las familias de sectores populares. Es necesario comprender que la escuela es importante para muchas familias de sectores populares, pero el mito de la escuela como progreso necesita renovarse. Es necesario entender a qué tipo de progreso apuntan las familias o, en todo caso, cómo la escuela es necesaria para este proyecto. Se trata, entonces, de comprender cuál es el valor que hoy tiene la escuela para la juventud, las y los niños, las y los adultos, y las familias en general. ¿Qué tipo de escuela sería capaz de potencializar la construcción de estrategias de las familias de los sectores populares en torno a la escuela?

Esta reflexión no debe perder de vista que el Estado es responsable por la gratuidad de la educación básica regular, por la calidad de la educación e incluso por la adaptación del sistema escolar a las condiciones económicas y culturales de los espacios donde se encuentra (MED, 2007). Se trata, pues, de garantizar el cumplimiento de la normativa ya existente, como la adaptación del calendario escolar en áreas rurales donde los y las jóvenes trabajan, y de mejorar el sistema de educación pública. Es en esa mejoría del sistema, del servicio ofrecido, para la satisfacción del derecho, que es importante conocer cuáles son las necesidades y los intereses de las familias, y conocer por qué esperamos, como país, que estas apuesten por la escuela y por la validez de la educación pública. 


\section{Referencias}

Alves, N. (2006). Socialização escolar e profissional dos jovens: projetos, estratégias e representações. Cadernos Sísifo 1. Lisboa: Educal, Unidade de I\&D de ciências da educação.

Ansión, J. (1989). La escuela en la comunidad campesina. Proyecto Escuela, Ecología y Comunidad Campesina. Lima: FAO-Suiza y Ministerio de Agricultura.

Bourdieu, P. \& Passeron, J.-C. (1998). La reproducción. Elementos para una teoría del sistema de enseñanza. México: Fontamara.

Bourdieu, P. \& Passeron, J.-C. (2004). Los herederos. Los estudiantes y la cultura. Buenos Aires: Siglo XXI.

Carabaña, J. (2002). Las políticas de izquierda y la igualdad educativa. En J. Torreblanca (Ed.), Los fines de la educación. Una reflexión desde la izquierda (pp. 169-225). Madrid: Biblioteca Nueva.

Carvalho, M. P., de (2003). Sucesso e fracasso escolar: uma questão de gênero. Educação e Pesquisa, 29(1), 185-193.

Charlot, B. (1996). Relação com o saber e com a escola entre estudantes de periferia. Cadernos de pesquisa, 97, 47-63.

Coleman, J. S., Campbell, E. Q., Hobson, C. J., McPartland, J., Mood, A. M., Weinfeld, F. D. \& York, R. L. (1966). Equality of educational opportunity. Washington: USA Department of Health, Education and Welfare.

Dayrell, J. (1996). A escola como espaço sócio-cultural. Múltiplos olhares sobre educação e cultura, 136-161. Belo Horizonte: UFMG.

Degregori, C. I. (1986). Del mito del Inkarri al mito del progreso: poblaciones andinas, cultura e identidad nacional. Socialismo y participación, 36, 48-56. Lima: Centro de Estudios para el Desarrollo y la Participación (CEDEP).

Diez, A. (1998). Creación de escuelas, mitos y cambios culturales en la sierra de Piura. Revista Antropológica, año XVI, 16, 131-148. Lima: Pontificia Universidad Católica del Perú.

Dubet, F. \& Martuccelli, D. (1998). En la escuela. Sociología de la experiencia escolar. Buenos Aires: Losada. 
Durkheim, E. (1998). Educación y pedagogía. Ensayos y controversias. Buenos Aires: Losada (Biblioteca pedagogía).

Epstein, J. (2005). Involvement counts: Family and community partnerships and mathematics achievement. The Journal of Educational Research, 98, 196-206.

Giroux, H. (1997). Teoría y resistencia en educación. México D.F.: Siglo XXI.

Guedes, S. L. (1997). Jogo de corpo. Um estudo de construção social de trabalhadores. Niteori: UFF.

Lahire, B. (1997). Sucesso escolar nos meios populares. As razões do impossível. São Paulo: Ática.

Lareau, A. (2002). Invisible inequality: Social class and childrearing in black families and white families. American Sociological Review, 67, 747-776.

McLaren, P. (1995). La escuela como performance ritual. Hacia una economía política de los símbolos y gestos educativos. México D.F.: Siglo XXI.

Mickelson, R. A. (2003). Gender, Bourdieu and the anomaly of women's achievement redux. Sociology of Education. 76(4), 373-375.

Ministerio de Educación del Perú. (2006). Directiva para el año escolar 2007. Normas y orientaciones nacionales para la gestión de las instituciones educativas de educación básica y educación técnico-productiva. Recuperado de http://www.educared.pe/modulo/upload/122747894. pdf

Ministerio de Educación del Perú. (2007). Diseño Curricular Nacional de la Educación Básica Regular (EBR). Lima: MINEDU.

Montero, C. (1995). Ciclos de vida y tiempos de escuela: el caso de las mujeres en el Perú. En S. C. Bourque, C. Montero \& T. Tovar (Eds.), ¿'Todos igualitos? Género y educación (pp. 43-66). Lima: Pontificia Universidad Católica del Perú.

Montoya, R. (1980). Capitalismo y no capitalismo en el Perú. Un estudio histórico de su articulación en un eje regional. Lima: Mosca Azul.

Nogueira, M. E. (2000). Família e escola. Trajetórias de escolarização em camadas médias e populares. Petrópolis: Vozes.

Olivera, I. (2005). Diferencias entre hombres y mujeres en relación a la organización familiar y las oportunidades educativas en la familia campesina 
de Chaquira: el caso de un caserío de la costa piurana. (Tesis de Licenciatura). Pontificia Universidad Católica del Perú, Lima.

Olivera, I. (2008). Relação juventude-escola frente aos processos excludentes: discutindo as experiências sociais e os sentidos da escolaridade em Chaquira, um caserío rural no litoral norte do Peru. (Tesis de maestría). Universidade Federal de Santa Catarina, Santa Catarina.

Olivera, I. (2009). Los sentidos de la escolaridad. O la relación juventud ruralescuela frente a los procesos de exclusión. Revista Peruana de Investigación Educativa, 1(1), 61-90.

Parsons, T. (1959). The school class as a social system: Some of its functions in American society. Social structure and personality. London: McMillan.

Reimers, F. \& Villegas-Reimers, E. (2006). Sobre la calidad de la educación y su sentido democrático. Revista PRELAC, 2, 90-107.

Rosemberg, F. (2001). Educação formal, mulher e gênero no Brasil contemporâneo. Revista de Estudos Feministas, 9(2), 515-539.

Thin, D. (2006). Para uma analise das relações entre famílias populares e escola: confrontações entre lógicas socializadoras. Revista Brasileira de educação, 11(32), 211-370.

Zago, N. (2000). Processos de escolarização nos meios populares. As contradições da obrigatoriedade escolar. En M. A. Nogueira, G. Romanelli \& N. Zago (Orgs.), Família e escola. Trajetórias de escolarização em camadas médias e populares (pp. 17-73). Petrópolis: Vozes.

Zoomers, A. (Ed.) (1998). Estrategias campesinas: algunas consideraciones teóricas y conceptuales. Estrategias campesinas en el surandino de Bolivia: intervenciones y desarrollo rural en el norte de Chuquisaca y Potosí. La Paz: CEDLA-Pluras Editores. 